\title{
Pruritic Axillary Plaques
}

Leah K. Spring, DO; Alexander J. Pybus, MD; Michael L. Crandall, MD

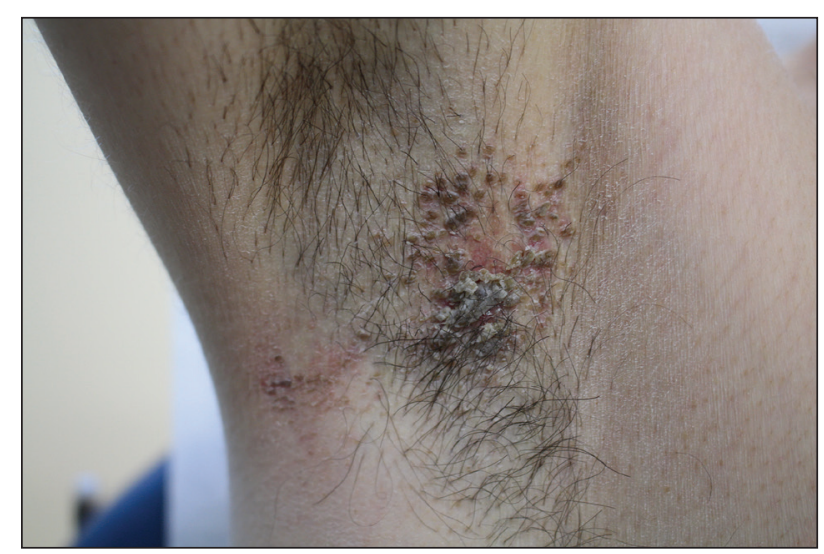

A 42-year-old man presented with pruritic axillary plaques of 6 months' duration that were exacerbated by heat and friction. He maintained a very active lifestyle and used an antiperspirant regularly. He denied any family history of similar lesions. Thick emollients provided no relief. Physical examination demonstrated numerous soft, hyperkeratotic, waxy, yellowish brown papules coalescing into plaques localized to the bilateral axillary vaults, affecting the right axilla more than the left. Although some papules were firmly adherent to the skin, others were friable and easily removed with a cotton-tipped applicator, revealing an underlying, faintly erythematous base.

\section{WHAT'S THE DIAGNOSIS?}

\author{
a. allergic contact dermatitis \\ b. granular parakeratosis \\ c. Hailey-Hailey disease \\ d. inverse psoriasis \\ e. keratosis follicularis (Darier disease)
}




\section{THE DIAGNOSIS:}

\section{Granular Parakeratosis}

$\mathrm{M}$

icroscopic examination of a punch biopsy from the left axilla revealed verruciform epidermal hyperplasia with overlying parakeratosis and retention of keratohyalin granules in the stratum corneum (Figure). There was no evidence of acantholysis, dyskeratosis, epidermal neutrophils, or neutrophilic microabscesses.

The patient's history and characteristic histopathologic findings confirmed the diagnosis of granular parakeratosis (GP). He was instructed to discontinue his current antiperspirant and began treatment with topical fluocinolone oil $0.01 \%$ every morning and urea cream $20 \%$ every night. Complete resolution was achieved within 2 weeks, and he reported no recurrence at a 2 -year follow-up visit.

Granular parakeratosis is a rare idiopathic skin condition characterized by hyperkeratotic papules and plaques, most often in intertriginous areas. Described by Northcutt et $\mathrm{al}^{1}$ as a contact reaction to antiperspirant in the axillae, GP also has been reported in the submammary and inguinal creases ${ }^{2}$ and rarely in nonintertriginous sites such as the abdomen. ${ }^{3}$ Although deodorants and antiperspirants in roll-on or stick form classically are implicated in GP, the condition also has been observed with exposure to laundry detergents containing benzalkonium chloride. ${ }^{4}$ Lesions with GP histology also have been incidentally observed in association with dermatophytosis, ${ }^{5}$ dermatomyositis, ${ }^{6}$ molluscum contagiosum, ${ }^{7}$ and carcinomas. ${ }^{8}$ Ding et $\mathrm{al}^{9}$ proposed that GP be reclassified as a reaction pattern observed in the skin as opposed to being a distinct disease entity.

Clinically, GP presents as pruritic intertriginous papules and coalescent plaques that most commonly are seen in the axillae but also may involve the groin or other sites. ${ }^{2,3}$ Both pruritus and disease burden can be aggravated by heat, sweating, or friction. There may be a history of a new irritant exposure prior to symptom onset, but GP has been observed in the absence of identifiable exposures and in the setting of long-term antiperspirant or deodorant use. ${ }^{3}$ Although a family history may be helpful, it can be difficult to distinguish GP from entities such as Hailey-Hailey disease or Darier disease based on history and examination alone; a biopsy often is necessary for definitive diagnosis.

Histologically, GP demonstrates acanthosis with parakeratosis and retention of keratohyalin granules in the stratum corneum. ${ }^{1}$ The stratum granulosum is preserved. On cursory examination, GP may resemble a psoriasiform dermatosis as can be seen in inverse psoriasis; however, neutrophilic microabscesses and infiltrates are not seen. Absence of acantholysis and dyskeratosis further differentiates GP from the clinically similar Hailey-Hailey disease

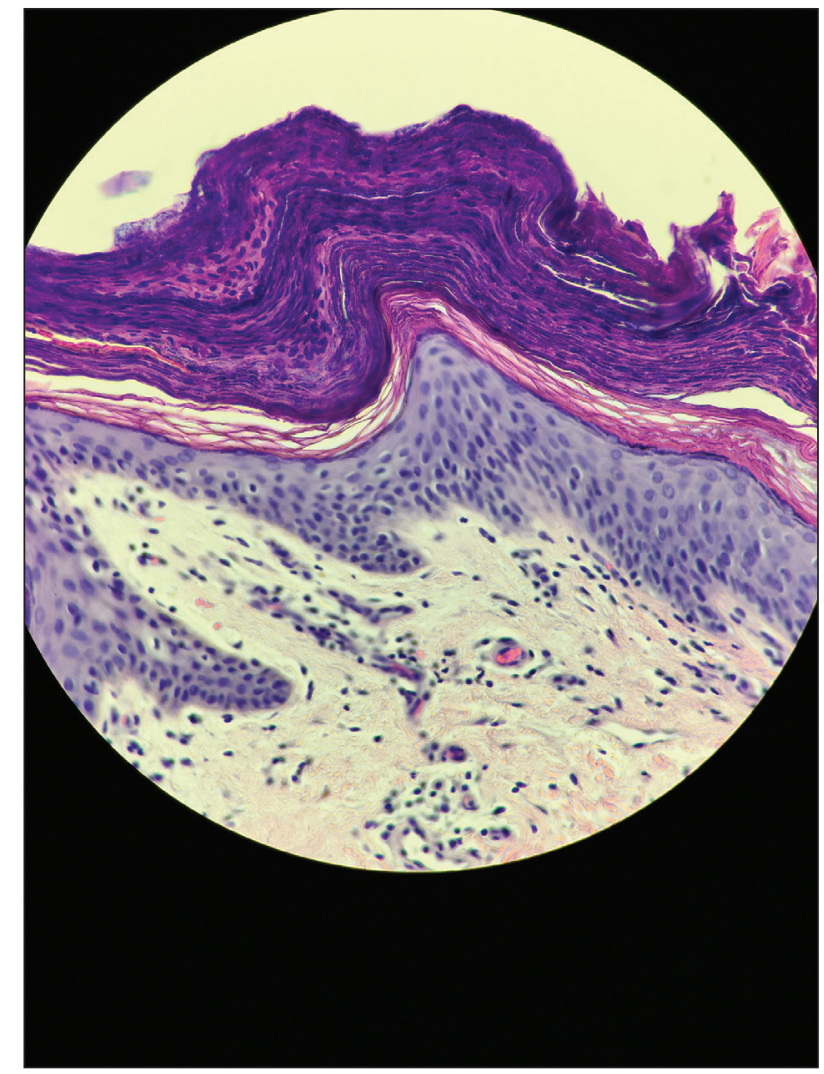

Verruciform epidermal hyperplasia with overlying parakeratosis and retention of keratohyalin granules in the stratum corneum (H\&E, original magnification $\times 100$ )

and Darier disease. Spongiosis that is prominently found in allergic contact dermatitis also is absent.

Although a benign disorder, GP warrants treatment to achieve symptomatic relief. A mainstay of treatment is to eliminate exposure to suspected aggravating or inciting factors such as antiperspirants or deodorants. A variety of treatments including laser therapy, corticosteroids, isotretinoin, and vitamin D analogs such as calcipotriene and calcitriol have been reported to be effective treatments of GP in case studies and series. ${ }^{3,10}$ Large-scale clinical trials are not available because of the rarity of this condition. Our patient's clinical course suggests topical fluocinolone and urea in combination can be considered to achieve rapid resolution.

\section{REFERENCES}

1. Northcutt AD, Nelson DM, Tschen JA. Axillary granular parakeratosis. J Am Acad Dermatol. 1991;24:541-544

2. Burford C. Granular parakeratosis of multiple intertriginous areas. Australas J Dermatol. 2008;49:35-38 
3. Samrao A, Reis M, Neidt G, et al. Granular parakeratosis: response to calcipotriene and brief review of current therapeutic options. Skinmed. 2010;8:357-359.

4. Robinson AJ, Foster RS, Halbert AR, et al. Granular parakeratosis induced by benzalkonium chloride exposure from laundry rinse aids. Australas J Dermatol. 2017;58:E138-E140.

5. Resnik KS, Kantor GR, DiLeonardo M. Dermatophyte-related granular parakeratosis. Am J Dermatopathol. 2004;26:70-71.

6. Pock L, Hercogová J. Incidental granular parakeratosis associated with dermatomyositis. Am J Dermatopathol. 2006;28:147-149.
7. Pock L, Cermáková A, Zipfelová J, et al. Incidental granular parakeratosis associated with molluscum contagiosum. Am J Dermatopathol. 2006;28:45-47.

8. Resnik KS, DiLeonardo M. Incidental granular parakeratotic cornification in carcinomas. Am J Dermatopathol. 2007;29:264-269.

9. Ding CY, Liu H, Khachemoune A. Granular parakeratosis: a comprehensive review and a critical reappraisal. Am J Clin Dermatol. 2015; 16:495-500.

10. Patel U, Patel T, Skinner RB. Resolution of granular parakeratosis with topical calcitriol. Arch Dermatol. 2011;147:997-998. 